\title{
Effects of neurally adjusted ventilatory assist on air distribution and dead space in patients with acute exacerbation of chronic obstructive pulmonary disease
}

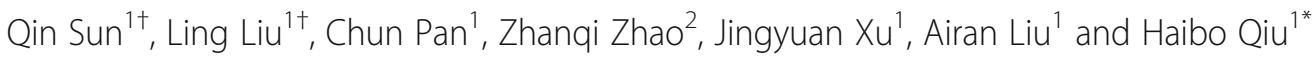

\begin{abstract}
Background: Neurally adjusted ventilatory assist (NAVA) could improve patient-ventilator interaction; its effects on ventilation distribution and dead space are still unknown. The aim of this study was to evaluate the effects of varying levels of assist during NAVA and pressure support ventilation (PSV) on ventilation distribution and dead space in patients with acute exacerbation of chronic obstructive pulmonary disease (AECOPD).

Methods: Fifteen mechanically ventilated patients with AECOPD were included in the study. The initial PSV levels were set to $10 \mathrm{cmH}_{2} \mathrm{O}$ for $10 \mathrm{~min}$. Thereafter, the ventilator mode was changed to NAVA for another 10 min with the same electrical activity of the diaphragm as during PSV. Furthermore, the ventilation mode was switched between PSV and NAVA every 10 min in the following order: PSV $5 \mathrm{cmH}_{2} \mathrm{O}$; NAVA 50\%; PSV $15 \mathrm{cmH}_{2} \mathrm{O}$; and NAVA $150 \%$ (relative to the initial NAVA support level). Ventilation distribution in the lung was evaluated in percentages in regions of interest $(\mathrm{ROI})$ of four anteroposterior segments of equal height ( $\mathrm{ROI} 1$ to $\mathrm{RO} 4$ represents ventral, midventral, mid-dorsal, and dorsal, respectively). Blood gases, ventilation distribution (electrical impedance tomography), diaphragm activity (B-mode ultrasonography), and dead space fraction $\left(\mathrm{PeCO}_{2}\right.$ and $\left.\mathrm{PaCO}_{2}\right)$ were measured.

Results: The trigger and cycle delays were lower during NAVA than during PSV. The work of trigger was significantly lower during NAVA compared to PSV. The diaphragm activities based on ultrasonography were higher during NAVA compared to the same support level during PSV. The ventilation distribution in ROI4 increased significantly $(P<0.05)$ during NAVA compared to PSV (except for a support level of 50\%). Similar results were found in ROI3+4. NAVA reduced dead space fraction compared to the corresponding support level of PSV.
\end{abstract}

Conclusions: NAVA was superior to PSV in AECOPD for increasing ventilation distribution in ROI4 and reducing dead space.

Trial registration: Clinicaltrials.gov, NCT02289573. Registered on 12 November 2014.

Keywords: Intratidal gas distribution, Vd/Nt, Patient-ventilator interaction, Work of trigger, Electrical impedance tomography

\footnotetext{
* Correspondence: haiboq2000@163.com

${ }^{\dagger}$ Equal contributors

'Department of Critical Care Medicine, Zhongda Hospital, School of Medicine, Southeast University, No.87, Dingjiagiao Road, Gulou District, Nanjing 210009, Jiangsu, China

Full list of author information is available at the end of the article
} International License (http://creativecommons.org/licenses/by/4.0/), which permits unrestricted use, distribution, and reproduction in any medium, provided you give appropriate credit to the original author(s) and the source, provide a link to the Creative Commons license, and indicate if changes were made. The Creative Commons Public Domain Dedication waiver (http://creativecommons.org/publicdomain/zero/1.0/) applies to the data made available in this article, unless otherwise stated. 


\section{Background}

The acute exacerbation of chronic obstructive pulmonary disease (AECOPD) is a disease state characterized by expiratory airflow limitation, increasing dead space, and diaphragm dysfunction [1], and invasive mechanical ventilation (MV) is required in the presence of severe respiratory failure [2]. Earlier studies have found less ventilation distribution in the near-diaphragm region and more dead space in patients with chronic obstructive pulmonary disease (COPD) [3, 4]. Improving heterogeneous ventilation distribution and decreasing dead space are important during MV in AECOPD patients [3, 4].

Pressure support ventilation (PSV), an MV mode with constant pressure assistance, is commonly used in patients with AECOPD; however, PSV has been proven to intensify heterogeneous ventilation distribution in patients with acute lung injury (ALI) [5]. Neurally adjusted ventilatory assist (NAVA) is a new MV mode that is triggered using diaphragm electrical activity (EAdi) and gives the amount of pressure assistance that is proportional in relation to EAdi. In theory, NAVA can improve heterogeneous ventilation distribution by decreasing the contraction of diaphragm, which Paul Blankman and his colleagues have proven [5].

Patients with AECOPD also suffer from increasing dead space and decreasing ventilation efficiency [4]. Over-assistance during PSV may exacerbate this problem [6]. NAVA provides the amount of pressure assistance in relation to EAdi and reduces the risk of over-assistance due to downregulation of the EAdi signal [7-13], which may be a solution to increasing dead space during MV in patients with AECOPD.

Based on this analysis, we designed a study to distinguish the difference between NAVA and PSV on ventilation distribution and dead space in patients with AECOPD. We hypothesized that NAVA could be a better MV mode for patients with AECOPD by improving heterogeneous ventilation distribution and decreasing dead space.

\section{Methods}

\section{Study population}

All study patients or their family members signed informed consent forms. The protocol was approved by the local institutional human investigations committee (IEC for Clinical Research of Zhongda Hospital, Affiliated to Southeast University, 2014ZDSYLL086.0). Patients who were admitted to the intensive care unit (ICU), intubated because of AECOPD, and ventilated by PSV were eligible for inclusion in the study. The exclusion criteria were as follows: (1) age <18 years or > 85 years; (2) ventilated because of oesophageal surgery in the previous 12 months; (3) oesophageal bleeding in the previous 30 days; (4) history of oesophageal varices; (5) facial trauma and surgery; (6) haemodynamic instability despite adequate filling (i.e. the need for continuous infusion of epinephrine or vasopressin, or dopamine $>5 \mu \mathrm{g} / \mathrm{kg} / \mathrm{min}$ or norepinephrine $>0.1 \mu \mathrm{g} / \mathrm{kg} / \mathrm{min}$ to maintain systolic arterial blood pressure >90 mmHg); (7) coagulation disorders (international normalised ratio $($ INR) $>1.5$ and partial thromboplastin time (PTT) $>44 \mathrm{~s}$ ); and (8) inclusion in other research protocols.

\section{Experimental protocol}

At the time of enrolment, all patients were mechanically ventilated using PSV and Servo-I (Maquet, Solna, Sweden). The standard nasogastric tubes of all included patients were replaced by the modified NAVA tube positioned according to the manufacturer's recommendations [7]. Airway suctioning was performed adequately before the beginning of the protocol. There was no airway suctioning during the protocol. During the entire recording period, positive end-expiratory pressure (PEEP), fraction of inspired oxygen $\left(\mathrm{FiO}_{2}\right)$, inspiratory trigger, and cyclingoff settings were maintained as set by the clinician in charge of the patient. The initial PSV levels were set to 10 $\mathrm{cmH}_{2} \mathrm{O}$ (denoted as PSV 100) for $10 \mathrm{~min}$. Thereafter, the ventilator mode was changed to NAVA for another 10 min. The EAdi during PSV 100 was used to titrate to the corresponding initial NAVA gain (NAVA100). Furthermore, the ventilation mode switched between PSV and NAVA every $10 \mathrm{~min}$ in the following order: PSV of 5 $\mathrm{cmH}_{2} \mathrm{O}$ (PSV50); NAVA 50\% support level of NAVA100 (NAVA50); PSV of $15 \mathrm{cmH}_{2} \mathrm{O}$ (PSV150); and NAVA 150\% support level of NAVA100 (NAVA150).

The electrical impedance tomography (EIT) measurements were performed using a 16-electrode silicon belt placed around the patient's thoracic cage just below the nipples and between the 4th and 5th intercostal spaces (Pulmovista 500, Dräger Medical, Lübeck, Germany). The data were gathered at a sampling frequency of $20 \mathrm{~Hz}$. Ventilation distribution was evaluated in percentages in regions of interest (ROI) of four anteroposterior segments of equal height [14]. ROI1 corresponded to the most non-dependent regions, whereas ROI4 denoted the most dependent regions. The regional ventilation-delay index estimating the early and late opening of lung tissues was calculated according to a previous study [15, 16]. The ventilator waveform was recorded using a Servo-I ventilator, and all signals were digitized at $100 \mathrm{~Hz}$ and were stored for offline analysis (NeuroVent Research Inc., Toronto, ON, Canada).

Two minutes before changing the ventilator mode, the diaphragm activity was measured using B-mode ultrasonography (FUJiFILM SonoSite Inc.). The ultrasound images of the right hemidiaphragm were obtained using a mechanical sector scanner fitted with a $5-\mathrm{MHz}$ transducer (Honeywell Ultraimager). The transducer was placed below the right costal margin and, with the liver 
providing an acoustic window, saggital views of the posterior portion of the diaphragm were obtained until a maximum diaphragmatic excursion was observed. The transducer was held at the described position and the movement of the right hemidiaphragm was recorded as an M-mode tracing. Five consecutive respiratory cycles were measured, and the average movement was recorded as diaphragm activity.

The dead space fraction $(\mathrm{Vd} / \mathrm{Vt})$ was estimated based on the following equation: $\mathrm{Vd} / \mathrm{Vt}=\left(\mathrm{PaCO}_{2}-\mathrm{PeCO}_{2}\right)$ / $\mathrm{PaCO}_{2}$,

where $\mathrm{PaCO}_{2}$ is obtained from the arterial blood ventilation measurements and $\mathrm{PeCO}_{2}$ is the mixed expired $\mathrm{PCO}_{2}$ [17]. A Douglas bag was attached to the exhalation port of the ventilator, and the $\mathrm{PeCO}_{2}$ was collected over $15 \mathrm{~min}$. At the end of the timed collection, $\mathrm{PeCO}_{2}$ of the ventilation collected in the Douglas bag was analysed using a blood ventilation analyser (Radiometer, Westlake, $\mathrm{OH}, \mathrm{USA}$ ). $\mathrm{PaO}_{2}$ and $\mathrm{PaCO}_{2}$ were measured after each step.

\section{Statistics}

Before starting the protocol, we calculated the sample size using the following formula: $n=2 \times((\alpha+\beta) \sigma / \delta)^{2}$, and the minimum sample size was 13 . The statistical analyses were performed using SPSS 20 (Chicago, IL, USA). The values are shown as the mean \pm SD unless specified otherwise. For the normally distributed data, paired samples $t$ tests and Bonferroni correction were used. We analysed the data that did not meet the assumption of normality using the means of nonparametric statistical tests. The correlation of diaphragm activity and ventilation distribution in ROI 4 was evaluated using Pearson two-tailed test. The differences were considered to be significant when $P<0.05$.

The primary endpoint of the study was to investigate changes in ventilation distribution and $\mathrm{Vd} / \mathrm{Vt}$ between NAVA and PSV. Our secondary endpoints included diaphragm activity, regional compliance, and regional ventilation delay. Because ventilation distribution data and regional ventilation delay did not meet the assumption of normality, we analysed them using non-parametric statistical tests. In this study, we also investigated the changes in ventilation distribution in gravity-dependent lung regions, dead space fraction between NAVA and PSV and diaphragm activity. Additionally, we analysed the differences in EAdi, peak pressure, respiratory rate, mean inspiratory pressure, expiratory tidal volume $(\mathrm{Vt})$, volume, trigger delay (in seconds), cycle delay (in seconds), work of breathing (in $\mu \mathrm{V} \cdot \mathrm{s}$ ) and work of trigger (in $\mu \mathrm{V} \cdot \mathrm{s}$ ) between PSV and NAVA. For both PSV 100 and NAVA 100, there were no significant differences between peak EAdi.

\section{Results}

Fifteen patients with AECOPD were admitted to this study from January 2015 to February 2016. The entry characteristics of the study population are presented in Table 1.

Table 1 Main characteristics of patients at inclusion

\begin{tabular}{|c|c|c|c|c|c|c|c|}
\hline Patient & Sex & Age (years) & APACHE ॥ & SOFA & Cause of AECOPD & PEEPi $\left(\mathrm{cmH}_{2} \mathrm{O}\right)$ & Ventilation time (days) \\
\hline 1 & $M$ & 85 & 15 & 10 & Sepsis & 6 & 4 \\
\hline 2 & M & 79 & 10 & 5 & Sepsis & 4 & 9 \\
\hline 3 & $\mathrm{~F}$ & 67 & 12 & 6 & Sepsis & 3 & 5 \\
\hline 4 & M & 85 & 15 & 12 & Sepsis & 8 & 15 \\
\hline 5 & M & 83 & 14 & 12 & Sepsis & 3.6 & 19 \\
\hline 6 & M & 74 & 15 & 11 & Sepsis & 4 & 16 \\
\hline 7 & M & 85 & 25 & 9 & Sepsis & 4 & 5 \\
\hline 8 & M & 79 & 24 & 6 & Sepsis & 4 & 5 \\
\hline 9 & M & 84 & 23 & 10 & Sepsis & 4.2 & 5 \\
\hline 10 & M & 81 & 24 & 12 & Sepsis & 5 & 6 \\
\hline 11 & M & 73 & 18 & 7 & Sepsis & 3.6 & 3 \\
\hline 12 & M & 76 & 13 & 3 & Sepsis & 4 & 10 \\
\hline 13 & $\mathrm{~F}$ & 62 & 21 & 9 & Sepsis & 3 & 10 \\
\hline 14 & M & 82 & 24 & 12 & Sepsis & 2 & 3 \\
\hline 15 & M & 84 & 23 & 10 & Sepsis & 4.2 & 4 \\
\hline \multicolumn{2}{|c|}{ Mean \pm SD } & $78.6 \pm 7.01$ & $18.4 \pm 5.22$ & $8.93 \pm 2.89$ & & $4.17 \pm 1.39$ & $7.93 \pm 5.11$ \\
\hline
\end{tabular}


Table 2 Respiratory parameters varying pressure support and NAVA assist levels

\begin{tabular}{|c|c|c|c|c|c|c|}
\hline \multirow[t]{2}{*}{ Ventilator mode } & \multicolumn{2}{|l|}{$50 \%$} & \multicolumn{2}{|l|}{$100 \%$} & \multicolumn{2}{|l|}{$150 \%$} \\
\hline & PSV & NAVA & PSV & NAVA & PSV & NAVA \\
\hline Assist pressure $\left(\mathrm{cmH}_{2} \mathrm{O}\right)$ & $6.73 \pm 0.70$ & $6.88 \pm 1.08$ & $8.20 \pm 0.73^{b}$ & $7.68 \pm 1.39$ & $9.74 \pm 1.23^{c}$ & $7.94 \pm 1.25$ \\
\hline Ppeak $\left(\mathrm{cmH}_{2} \mathrm{O}\right)$ & $11.35 \pm 0.91$ & $12.88 \pm 3.30$ & $16.01 \pm 0.84$ & $16.52 \pm 4.82$ & $20.77 \pm 0.94$ & $18.81 \pm 4.29$ \\
\hline EAdi $(\mu \mathrm{V})$ & $14.69 \pm 8.72$ & $11.67 \pm 8.64$ & $11.48 \pm 7.75$ & $11.43 \pm 8.13$ & $14.33 \pm 6.87^{c}$ & $11.86 \pm 6.34$ \\
\hline Respiratory rate (/min) & $22.58 \pm 5.28$ & $22.34 \pm 4.58$ & $21.00 \pm 7.71$ & $23.06 \pm 5.40$ & $18.10 \pm 6.67$ & $19.59 \pm 5.52$ \\
\hline MVe (L/min) & $7.66 \pm 2.58$ & $7.39 \pm 2.20$ & $8.58 \pm 4.22$ & $8.41 \pm 3.32$ & $8.84 \pm 3.48^{c}$ & $7.65 \pm 3.00$ \\
\hline Inspiratory time (s) & $0.93 \pm 0.21$ & $1.02 \pm 0.14$ & $1.10 \pm 0.45$ & $1.01 \pm 0.21$ & $1.22 \pm 0.55$ & $1.05 \pm 0.18$ \\
\hline Vt $(L)$ & $0.35 \pm 0.09$ & $0.34 \pm 0.10$ & $0.42 \pm 0.14$ & $0.38 \pm 0.14$ & $0.53 \pm 0.21^{c}$ & $0.40 \pm 0.11$ \\
\hline Trigger delay (s) & $0.17 \pm 0.08^{\mathrm{a}}$ & $0.05 \pm 0.02$ & $0.25 \pm 0.23^{b}$ & $0.06 \pm 0.06$ & $0.34 \pm 0.18^{c}$ & $0.06 \pm 0.05$ \\
\hline Cycle delay (s) & $0.11 \pm 0.10^{a}$ & $0.02 \pm 0.01$ & $0.32 \pm 0.49^{b}$ & $0.06 \pm 0.11$ & $0.48 \pm 0.72^{c}$ & $0.05 \pm 0.07$ \\
\hline Work of breathing $(\mu \mathrm{V} \cdot \mathrm{s})$ & $7.31 \pm 5.76$ & $6.20 \pm 5.96$ & $6.10 \pm 5.83$ & $5.11 \pm 2.31$ & $7.33 \pm 4.13$ & $6.26 \pm 3.91$ \\
\hline Work of trigger $(\mu \mathrm{V} \cdot \mathrm{s})$ & $1.20 \pm 0.68^{\mathrm{a}}$ & $0.89 \pm 0.36$ & $1.52 \pm 1.25^{b}$ & $1.11 \pm 0.59$ & $2.17 \pm 1.08^{c}$ & $1.20 \pm 0.92$ \\
\hline$(\mathrm{ROI} 1+2) \times \mathrm{Vt}(\mathrm{L} / \mathrm{min})$ & $0.20 \pm 0.16^{a}$ & $0.17 \pm 0.13$ & $0.26 \pm 0.15$ & $0.20 \pm 0.14$ & $0.26 \pm 013^{c}$ & $0.21 \pm 0.14$ \\
\hline$(\mathrm{ROI} 3+4) \times \mathrm{Vt}(\mathrm{L} / \mathrm{min})$ & $0.21 \pm 0.12$ & $0.19 \pm 0.09$ & $0.20 \pm 0.12$ & $0.20 \pm 0.11$ & $0.24 \pm 0.12$ & $0.22 \pm 0.10$ \\
\hline
\end{tabular}

EAdi electrical activity of the diaphragm, MVe minute ventilatory volume, NAVA neutrally-adjusted ventilator assist, Ppeak peak pressure, PSV pressure support ventilation, $R O I$ regions of interest, $V t$ tidal volume

${ }^{a}$ Significant different versus NAVA $50 \%$

${ }^{\mathrm{b}}$ Significant different versus NAVA 100\%

'Significant different versus NAVA 150\%

The ventilatory data, including the levels of pressure support and the resulting EAdi values during each PSV and NAVA step, are shown in Table 2. There was no difference in assist pressure or tidal volume between PSV50 and NAVA50 (Table 2). By increasing the level of assist, tidal volume and mean inspiratory pressure increased, whereas the respiratory rate decreased during PSV and NAVA (Table 2). At the highest applied ventilatory assist level, tidal volume was significantly higher with PSV compared to NAVA (Table 2). At different support levels, trigger delay and cycle delay were lower during NAVA than during PSV.

The work of trigger was significantly lower during NAVA compared to PSV at three support levels, but there was no difference in the work of breathing (Table 2).

The diaphragm activity in patients with AECOPD decreased when the support level increased for both PSV and NAVA (Fig. 1). However, the diaphragm activities were higher during NAVA compared to the same support level during PSV (Fig. 1).

The ventilation distribution in ROI4 increased significantly $(P<0.05)$ during NAVA compared to PSV (except for a support level of 50\%). Similar results were found in ROI3 + 4. The ventilation distribution decreased in the ROI1 and ROI2 regions during NAVA (Table 3). The regional ventilation delay also varied in different lung regions. Four ROI had different ventilation times. We found that in the ROI4 region inspiration began earlier in NAVA compared to PSV (Fig. 2).
Higher levels of assist resulted in higher Vd/Vt values, and NAVA reduced $\mathrm{Vd} / \mathrm{Vt}$ values compared to the corresponding support level of PSV (Fig. 3). In this study, we also analysed the consistency of diaphragm activity and ventilation distribution in ROI4 and found that the correlation coefficient $R^{2}$ was $0.251(P<0.01)$ (Fig. 4).

\section{Discussion}

In this study, we found the following: (1) ventilation distribution in ROI4 increased significantly during NAVA

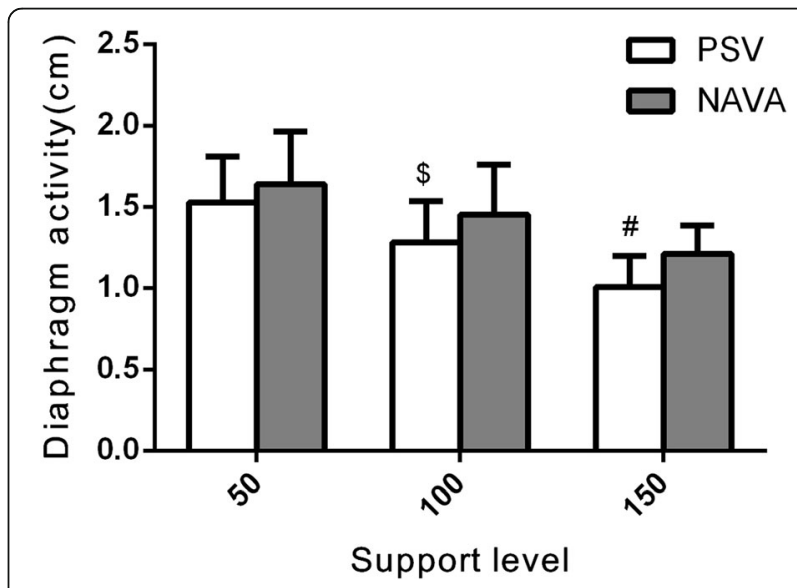

Fig. 1 Diaphragm activity in both PSV and NAVA. The bars represent the mean value and the lines represent the standard deviation. \$Significant differences versus NAVA 100\%; " significant differences versus NAVA 150\%. NAVA neutrally-adjusted ventilator assist, PSV pressure support ventilation 
Table 3 Gas distribution in four regions for both NAVA and PSV

\begin{tabular}{|c|c|c|c|c|c|c|c|c|c|}
\hline & \multicolumn{3}{|l|}{$50 \%$} & \multicolumn{3}{|l|}{$100 \%$} & \multicolumn{3}{|l|}{$150 \%$} \\
\hline & PSV & NAVA & P & PSV & NAVA & P & PSV & NAVA & P \\
\hline ROI 1 & $11.98 \pm 11.17$ & $11.76 \pm 10.65$ & 0.715 & $12.89 \pm 8.79$ & $11.86 \pm 8.89$ & 0.057 & $12.21 \pm 8.49$ & $11.11 \pm 9.37$ & $0.039^{a}$ \\
\hline $\mathrm{ROI} 2$ & $38.60 \pm 17.65$ & $36.36 \pm 18.71$ & 0.054 & $44.26 \pm 13.63$ & $39.02 \pm 15.07$ & $0.003^{\mathrm{a}}$ & $41.94 \pm 14.80$ & $37.58 \pm 13.87$ & $0.004^{a}$ \\
\hline ROI 3 & $39.79 \pm 21.54$ & $41.37 \pm 22.41$ & 0.220 & $35.75 \pm 18.50$ & $40.13 \pm 19.64$ & $0.014^{\mathrm{a}}$ & $37.58 \pm 18.82$ & $41.37 \pm 18.82$ & 0.18 \\
\hline ROI 4 & $9.66 \pm 8.42$ & $10.98 \pm 9.05$ & 0.158 & $7.53 \pm 4.98$ & $9.25 \pm 5.54$ & $0.005^{\mathrm{a}}$ & $9.10 \pm 6.71$ & $10.89 \pm 8.06$ & $0.020^{a}$ \\
\hline $\mathrm{ROI} 3+4$ & $49.46 \pm 27.28$ & $52.35 \pm 27.97$ & 0.093 & $43.28 \pm 20.22$ & $49.38 \pm 22.00$ & $0.007^{\mathrm{a}}$ & $46.68 \pm 21.94$ & $52.26 \pm 22.22$ & $0.004^{\circ}$ \\
\hline
\end{tabular}

NAVA neutrally-adjusted ventilator assist, PSV pressure support ventilation, $R O I$ regions of interest

${ }^{a}$ Significantly different versus NAVA

compared to PSV (except for a support level of 50\%), and similar results were found in $\mathrm{ROI} 3+4$; (2) diaphragm activities measured using ultrasonography were higher during NAVA compared to the same support level during PSV; and (3) NAVA reduced Vd/Vt values compared to the corresponding support level of PSV. Additionally, we found that, at different support levels, trigger delay and cycle delay were lower during NAVA than during PSV in patients with AECOPD. The work of trigger was significantly lower during NAVA compared to PSV at three support levels.

During NAVA, EAdi, an expression of the respiratory centre's activity, was used to trigger and cycle the ventilator, rather than a pneumatic signal located at the airway opening or inside the ventilator, which can significantly reduce trigger delay and cycle delay. Previous studies suggested that NAVA also decreased the work of trigger compared to PSV $[18,19]$. In PSV, the ventilator settings required a remarkable inspiratory effort to minimize the miss-triggering. In contrast with NAVA (when the ventilator's delivered cycle was triggered by the EAdi), as the ventilator's delivered pressurization immediately followed the increase in EAdi signal (without any depression on the pressure-time curve), less inspiratory effort was theoretically needed to trigger the ventilator. In our results, the work of trigger in NAVA was significantly lower than in PSV, and these results were consistent with findings in previous research. No significant differences were found in the work of breathing between NAVA and PSV.

Lowhagen and colleagues introduced the intertidal ventilation distribution based on EIT measurements during a trial consisting of ten patients with ALI [20]. They used the intertidal ventilation distribution to analyse how the tidal volume was distributed within the lung and found that the ventilation distribution in the ROI4

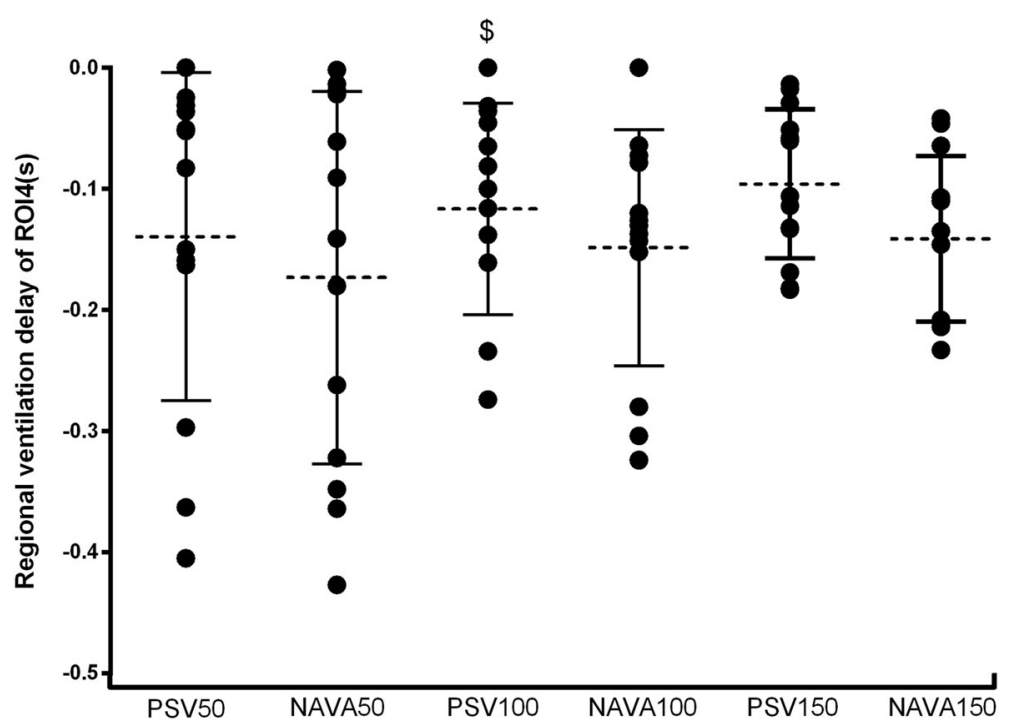

Fig. 2 Regional ventilation delay of ROI4 of every patient during a different ventilation mode. The dotted lines represent the medians and the solid lines represent quartiles. The negative value means that the ventilation began earlier than the null point. \$Significant differences versus NAVA 100\%. NAVA neutrally-adjusted ventilator assist, PSV pressure support ventilation, ROI regions of interest 


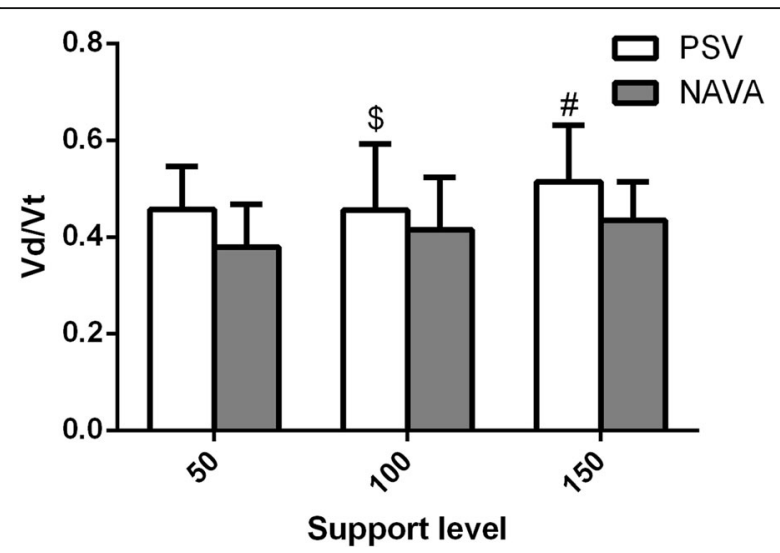

Fig. $3 \mathrm{Vd} / \mathrm{Nt}$ in both PSV and NAVA. The bars represent the mean values and the lines represent the standard deviation. ${ }^{\$}$ Significant differences versus NAVA 100\%; " significant differences versus NAVA 150\%. NAVA neutrally-adjusted ventilator assist, PSV pressure support ventilation

region increased during NAVA compared to PSV. In the present study, we found the same phenomenon in patients with AECOPD.

Many cross-sectional studies with careful matching of patients with controls have revealed the complexity of diaphragm dysfunction in COPD [21, 22]. Jérôme Cecchini and his colleagues found, that in critically ill and mechanically ventilated patients, increasing levels of PSV and NAVA reduced the diaphragm activity [23]. However, compared with PSV, NAVA resulted in a predominant contribution of the diaphragm to inspiratory effort. Our results showed a higher diaphragm activity during NAVA compared to PSV, and we found a correlation between diaphragm activity and ventilation distribution in ROI4. Furthermore, we found that in ROI4 inspiration began earlier in NAVA compared to PSV, which indicated an early onset of diaphragm contraction and a better patientventilator synchronisation with NAVA. Based on these results, we insist that NAVA could increase ventilation distribution in ROI4 by improving the diaphragm activity.

Dead space plays an important role in ventilation efficiency in patients with AECOPD, but it is difficult to measure directly. Therefore, we measured Vd/Vt instead of dead space. $\mathrm{Vd} / \mathrm{Vt}$ is a vital respiratory physiological index that represents the efficiency of lung ventilation [23]. We found that, in patients with AECOPD, Vd/Vt decreased significantly during NAVA. To explain this finding, we compared the tidal volume between PSV and NAVA and found that during NAVA the tidal volume was comparable despite the different levels of assist, whereas the tidal volume increased during increasing levels of PSV. This can be explained by the downregulation of the EAdi signal at higher assist levels during NAVA [7]. Additionally, we calculated the tidal volume distributed in ROI and $2((\mathrm{ROI} 1+2) \times \mathrm{Vt})$ and found that during NAVA the tidal volume distributed in ROI 1 and $2(($ ROI $1+2) \times V t)$ decreased significantly when compared to the same support level of PSV. This result meant that there was less ventilation distribution in the non-dependent lung region where pulmonary hyperinflation occurred easily. Improved ventilation distribution was another factor in the decreased Vd/Vt during NAVA.

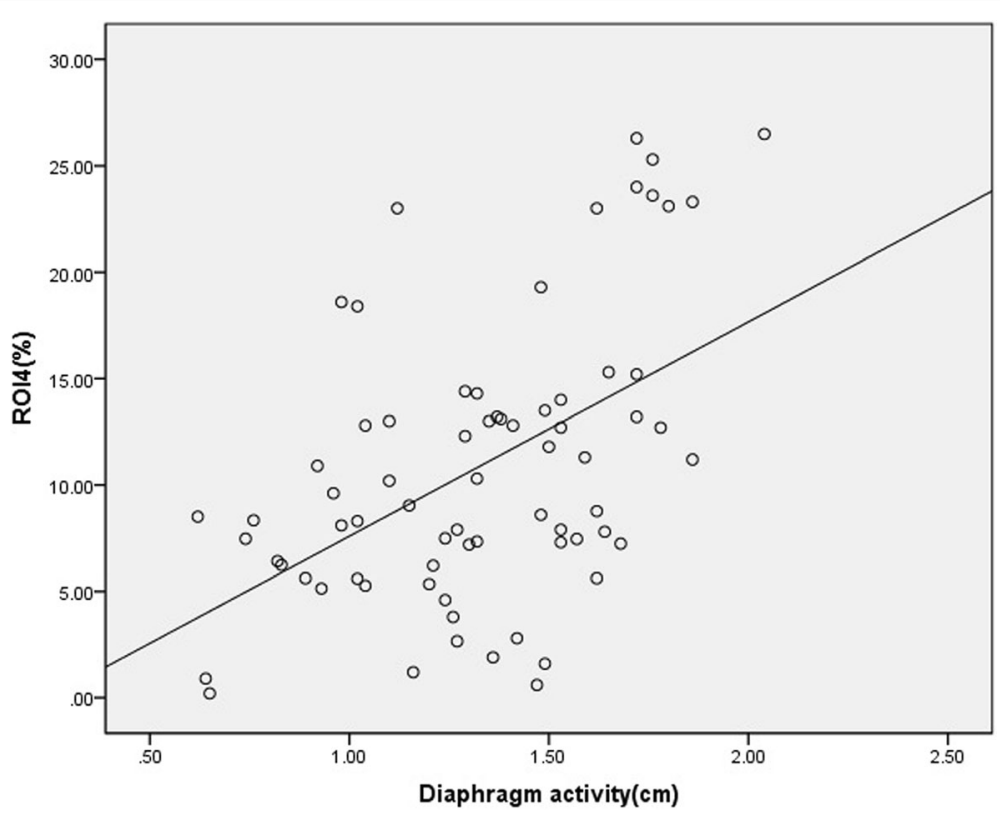

Fig. 4 Correlation between the diaphragm activity and ventilation distribution in $R O / 4 . R^{2}=0.56, P<0.01$. $R O /$ regions of interest 
There were several limitations in this study that must be addressed. One limitation of the study design was that the order of ventilation modes was not randomized. The results might be biased from the previous ventilator settings. Furthermore, the period of each ventilator setting was brief (10 $\mathrm{min})$, and there could be a risk that some physiologic variables could not reach their steady states within that period. The reason for those insignificant differences might be clarified in the findings between NAVA and PSV. Nevertheless, we identified significant differences in ventilation distribution, work of trigger, and $\mathrm{Vd} / \mathrm{Vt}$ values, which could be even more significant given a longer period of different settings. Even if the level of assistance in NAVA has been determined according to the usual procedure described by the manufacturer and reported in previous studies (NAVA gain or proportionality factor between EAdi and pressure delivered by the ventilator, set to obtain the same peak pressure in NAVA as in PSV), the comparability of assistance levels in PSV and NAVA is questionable. Other limitations included a one-centre design and small size. Further studies are needed.

\section{Conclusions}

NAVA can increase ventilation distribution in the neardorsal lung region and decrease $\mathrm{Vd} / \mathrm{Vt}$ value in patients with AECOPD. Furthermore, NAVA improves the patientventilator interaction in intubated patients with AECOPD and decreases the work of trigger. NAVA can be beneficial in this patient population. Additional studies should be conducted to determine whether NAVA could decrease ventilation time and improve outcome.

\section{Abbreviations \\ AECOPD: Acute exacerbations of chronic obstructive pulmonary disease; ALI: Acute lung injury; COPD: Chronic obstructive pulmonary disease; EAdi: Diaphragm electrical activity; EIT: Electrical impedance tomography; MV: Mechanical ventilation; NAVA: Neurally adjusted ventilatory assist; PEEP: Positive end-expiratory pressure; PSV: Pressure support ventilation; ROI: Regions of interest; Vt: Tidal volume}

\section{Acknowledgements}

This work was supported by the National Natural Science Foundation of China under the contract grant nos. 81300043 and 81300060 .

\section{Funding}

Not applicable.

\section{Availability of data and materials}

Not applicable.

\section{Authors' contributions \\ $H Q$, the corresponding author, was responsible for the concept, revision, and approval of this manuscript. QS and LL participated in the design and coordination of the study and drafted the manuscript. JX and AL were responsible for the data collection. CP and ZZ performed the statistical analysis and helped to revise the manuscript. All authors contributed to the data analysis and interpretation. All authors read and approved the final manuscript.}

\section{Competing interests}

The authors declare that they have no competing interests.

\section{Consent for publication}

All included patients or their family members signed the informed consent form, and they approved the publication of their individual details. The informed consent forms are held by the authors' institution and are available for review by the Editor-in-Chief if necessary. All authors have confirmed the manuscript and approved the publication of the manuscript. The corresponding author has completed the "Consent for publication".

\section{Ethics approval and consent to participate}

This study was approved by IEC for Clinical Research of Zhongda Hospital, Affiliated to Southeast University with a protocol number of 2014ZDSYLL086.0. Informed consent was provided by all study patients or their family members.

\section{Publisher's Note}

Springer Nature remains neutral with regard to jurisdictional claims in published maps and institutional affiliations.

\section{Author details}

${ }^{1}$ Department of Critical Care Medicine, Zhongda Hospital, School of Medicine, Southeast University, No.87, Dingjiaqiao Road, Gulou District, Nanjing 210009, Jiangsu, China. ${ }^{2}$ Institute of Technical Medicine, Furtwangen University, Villingen-Schwenningen, Germany.

Received: 25 November 2016 Accepted: 9 May 2017

Published online: 02 June 2017

References

1. Anna V, Matthew M, Dario M, et al. Muscle function in COPD: a complex interplay. Int J COPD. 2012;7:523-35.

2. Plant PK, Elliott MW. Chronic obstructive pulmonary disease 9: management of ventilatory failure in COPD. Thorax. 2003;58(6):537-42.

3. Simon BA, Kaczka DW, Bankier AA, Parraga G. What can computed tomography and magnetic resonance imaging tell us about ventilation? J Appl Physiol. 1985;113:647-57.

4. Gonzalea-Castro A, Suarez-Lopez V, Gomez-Marcos V, et al. Utility of the dead space fraction $(\mathrm{Vd} / \mathrm{Nt})$ as a predictor of extubation success. Med Intensiva. 2011;35(9):529-38.

5. Blankman P, Hasan D, van Mourik MS, et al. Ventilation distribution measured with EIT at varying levels of pressure support and neurally adjusted ventilatory assist in patients with ALI. Intensive Care Med. 2013; 39(6):1057-62.

6. Giannouli E, Webster K, Roberts D, et al. Response of ventilator-dependent patients to different levels of pressure support and proportional assist. Am J Respir Crit Care Med. 1999;159:1716-25.

7. Beck J, Campoccia F, Allo JC, et al. Improved synchrony and respiratory unloading by neurally adjusted ventilatory assist (NAVA) in lung-injured rabbits. Pediatr Res. 2007;61:289-94.

8. Colombo D, Cammarota G, Bergamaschi V, et al. Physiologic response to varying levels of pressure support and neurally adjusted ventilatory assist in patients with acute respiratory failure. Intensive Care Med. 2008:34:2010-8.

9. Oliva P, Schuffelmann C, GomezZamora A, et al. Asynchrony, neural drive, ventilator variability and COMFORT: NAVA versus pressure support in pediatric patients. A non-randomized cross-over trial. Intensive Care Med. 2012;38:838-46.

10. Piquilloud L, Vignaux L, Bialais E, et al. Neurally adjusted ventilator assist improves patient-ventilator interaction. Intensive Care Med. 2011;37:263-71.

11. Sinderby C, Beck J, Spahija J, et al. Inspiratory muscle unloading by neurally adjusted ventilatory assist during maximal inspiratory efforts in healthy subjects. Chest. 2007;131:711-7.

12. Spahija J, Marchie M, Albert $M$, et al. Patient-ventilator interaction during pressure support ventilation and neurally adjusted ventilatory assist. Crit Care Med. 2010;38:518-26.

13. Allo JC, Beck JC, Brander L, et al. Influence of neurally adjusted ventilatory assist and positive end-expiratory pressure on breathing pattern in rabbits with acute lung injury. Crit Care Med. 2006;34:2997-3004.

14. Pulletz S, Genderingen HR, Schmitz G, et al. Comparison of different methods to define regions of interest for evaluation of regional lung ventilation by EIT. Physiol Meas. 2006;27(5):S115-127.

15. Muders T, Luepschen $H$, Zinserling J, et al. Tidal recruitment assessed by electrical impedance tomography and computed tomography in a porcine model of lung injury. Crit Care Med. 2012;40(3):903-11. 
16. Costa EL, Borges JB, Melo A, et al. Bedside estimation of recruitable alveolar collapse and hyperdistension by electrical impedance tomography. Intensive Care Med. 2009;35(6):1132-7.

17. Rossier PH, Buhlmann A. The respiratory dead-space. Physiol Rev. 1955;35: $860-76$.

18. Hedenstierna $\mathrm{G}$, Tokics $\mathrm{L}$, Lundquist $\mathrm{H}$, et al. Phrenic nerve stimulation during halothane anesthesia.Effects of atelectasis. Anesthesiology. 1994;80: 751-60.

19. Ling $L$, Songqiao $L$, Jianfeng $X$, et al. Assessment of patient-ventilator breath contribution during neurally adjusted ventilatory assist in patients with acute respiratory failure. Crit Care. 2015;19(1):43.

20. Lowhagen K, Lundin S, Stenqvist O, et al. Regional intratidal ventilation distribution in acute lung injury and acute respiratory distress syndrome-assessed by electric impedance tomography. Minerva Anestesiol. 2010;76(12):1024-35.

21. Ottenheijm CA, Heunks LM, Sieck GC, et al. Diaphragm dysfunction in chronic obstructive pulmonary disease. Am J Respir Crit Care Med. 2005;172: 200-5.

22. Simon BA, Kaczka DW, Bankier AA. What can computed tomography and magnetic resonance imaging tell us about ventilation? J Appl Physiol (1985). 2012;113:647-57.

23. Cecchini J, Schmidt M, Demoule A, et al. Increased diaphragmatic contribution to inspiratory effort during neurally adjusted ventilatory assistance versus pressure support: an electromyographic study. Anesthesiology. 2014;121(5):1028-36.

\section{Submit your next manuscript to BioMed Central and we will help you at every step:}

- We accept pre-submission inquiries

- Our selector tool helps you to find the most relevant journal

- We provide round the clock customer support

- Convenient online submission

- Thorough peer review

- Inclusion in PubMed and all major indexing services

- Maximum visibility for your research

Submit your manuscript at www.biomedcentral.com/submit
C) Biomed Central 